\title{
Concentrated PhotoVoltaics (CPV): Is it a real opportunity?
}

\author{
P. Antonini \\ Centro Studi e Ricerche "E. Fermi" - P.zza Viminale, 1, 00184 Roma, Italy
}

\begin{abstract}
Summary. - Many large industries made relevant investment in the field of CPV. Considering the present price of traditional silicon cells one could argue about this strategy. Critical points of CPV together with its benefits will be presented. In CPV more technology is involved compared to PhotoVoltaic (PV) and this could be the key issue. Moreover, progress in the field of multiple-juction solar cells is constant and efficiency is increasing every year. Good technical solutions could make CPV very competitive, provided it is installed in areas where the direct light (DNI) is relevant.
\end{abstract}

\section{1. - Motivations}

Concentrated photovoltaics is based on the principle of concentrating sunlight on a smaller solar cell using cheap materials as molded plastics or aluminum to achieve a less expensive photovoltaics module. Moreover, using high-efficiency solar cells it is also possible to realize more efficient solar modules.

The main idea is simple: to concentrate solar light using cheap optical elements such as mirrors or lenses over much smaller solar cells (down to $1 / 1000$ ), and this to reduce the costs of installed power (and thus the costs of produced energy), see fig. 1 . Photovoltaics based on silicon solar cells need large areas, due to its low (about 14\%) efficiency. Concentrated photovoltaics can reach an efficiency near 30\%, thus it needs less land for the same installed power; silicon solar cells have a large (about -0.3\%) dependence of efficiency on temperature: this can be a real problem in very hot climates; 

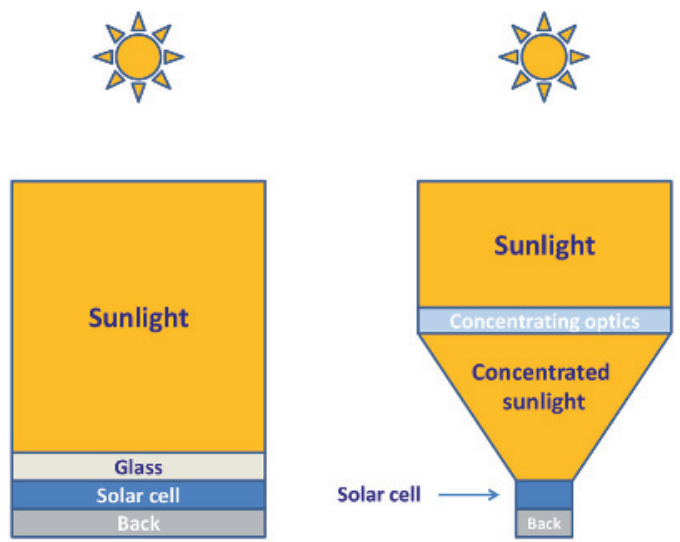

Fig. 1. - The idea behind concentrated photovoltaics is simple: replace expensive solar cells with cheap optical elements, e.g. molded plastic mirrors or lenses.

in opposite, 3J solar cells have a tenfold smaller coefficient. This is important because in CVP the rated power is nearer to the nominal one than in silicon PV.

The first large efforts in concentrating photovoltaics began in mid-1970s, pushed by the 1973 oil crisis [1]. The appearance in the market of price-affordable high-efficiency multiple junction solar cells about 30 years later added to this technology the value of using less land to generate the same amount of energy. The double-junction solar cells, with high efficiency (30\%) and above all the possibility to concentrate solar light to high levels, paved the way to the triple-junction solar cells, whose efficiency reached $40 \%$ in commercial products, and has overcome $43 \%$ in research cells.

Concentrated photovoltaics can be divided into four ranges, the borders can be set arbitrarily as:

- low concentration, between 2 and 10 times;

- medium concentration, between 10 and 100;

- high concentration, between 100 and 1000 times;

- very high concentration, over 1000 times.

Any concentrated photovoltaics module needs a solar tracker, a device that follows the path of the Sun during the day. For low concentrated photovoltaics a 1-axis Sun tracker may be used, whereas for higher concentrating ratios a dual axis Sun tracker must be used.

Notwithstanding this apparent simplicity, the expected high efficiency can be reached only mastering the several scientific and technological fields involved. Thus, the study of concentrated photovoltaics has as a result not only the prototype of an innovative module, but also an interdisciplinary knowledge useful in other applications, such as lighting techniques, LED, thermal solar (CSP) and hybrid automotive. 


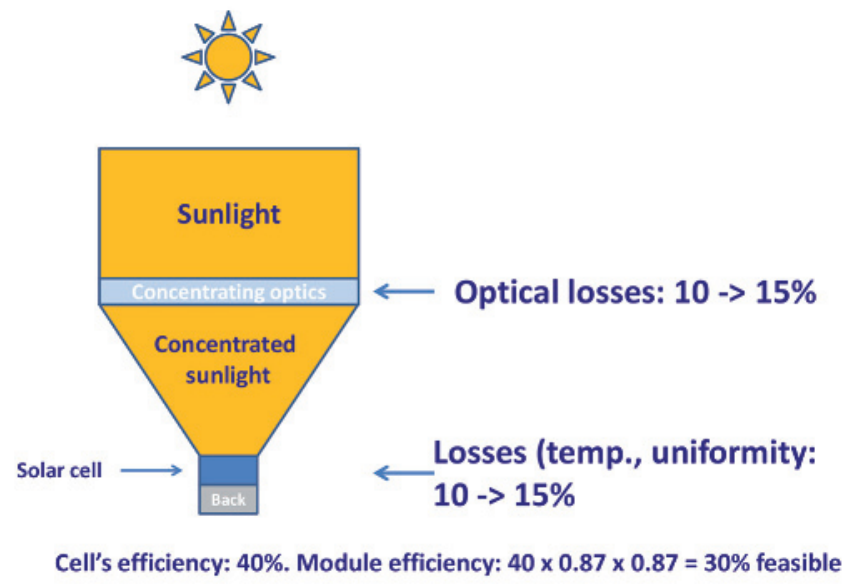

Fig. 2. - The use of high-efficiency (40\%) 3J solar cells allows to compensate for the losses of the concentrating optics and for the thermal and uniformity of irradiation losses of the cells. In principle a solar module with on-field efficiency of $30 \%$ or more is feasible.

1`1. Achievable efficiency. - As an example, consider fig. 2: the use of a $40 \%$ efficiency triple-junction solar cells allows to compensate for the losses and achieve a solar module with efficiency up to $30 \%$. In fact, the concentrating optics inevitably introduces some losses: reflection losses if it is a mirror, transmission losses if it is a lenses, both if it is a combination of mirror, lenses and homogenizers. With respect to losses, also aberrations play a role, as chromatic aberrations, coma, among others. The $3 \mathrm{~J}$ cell itself will not have the whole $40 \%$ of efficiency: this value is valid only for a cell at a temperature of $25^{\circ} \mathrm{C}$, and with uniform illumination. When this is not the case the efficiency of the cell will decrease. For a well-designed concentrator a value of $35 \%$ is reasonable. Thus the expected efficiency of the module should be, as an example, $40 \times 0.87 \times 0.87 \cong 30 \%$. To obtain this value then the module must be very well engineered, by making use of all the scientific and technological information.

In order for a CPV module to be commercially competitive, two criteria must be met: low cost of energy generated, and high reliability.

1`2. Different layouts. - The layouts of concentrating photovoltaics modules can be divided into three main structures (see fig. 3):

1) point focus;

2) linear focus;

3) heliostat.

Point focus photovoltaics is now the mainstream technology. It consists in concentrating optics that can be made of lenses, mirrors or combinations of them. The main characteristic is that the optics are folded around two directions (like a circular symmetry lens or 


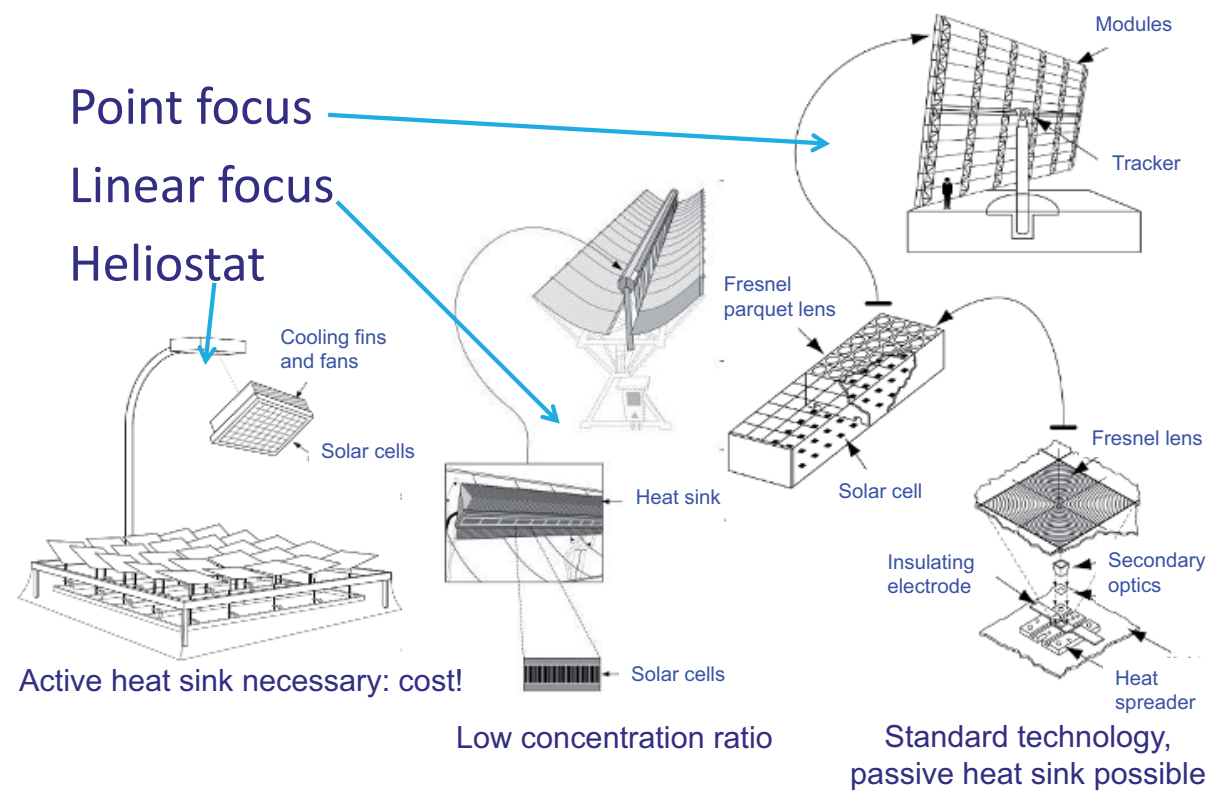

Fig. 3. - CPV systems are mainly of three different layouts. From left to right: heliostat, linear through, point focus. Images from [4].

parabola, but in practice the shapes are far more complicated), so that sunlight will be concentrated over distributed focuses. Two are the main advantages of this configuration: the possibility of higher concentration [2] and the possibility of passive heat sinking of the cells [3].

Linear focus concentrators are more used in solar thermal (CSP) applications. The main drawback being the difficulty to reach higher concentration: although in principle any concentration is geometrical possible, one is soon confronted with technological limits. A typical 3J solar cell has 3 to $10 \mathrm{~mm}$ dimension: a factor $500 \times$ concentration means a parabolic through 1.5 to $5 \mathrm{~m}$.

13. Optics. - To concentrate sunlight to the solar cells there are basically two approaches: using mirrors or using Fresnel lenses. There are some differences between these two technologies:

Using mirrors has the following advantages:

- mirrors do not have chromatic aberration: different wavelengths are diffracted to different angles;

- mirrors can be made of molded plastic, using the mature technology of automotive head lamps; 
- mirror itself can have the double function of optical component and the structural function of closing the module.

Using Fresnel lenses:

- the design of the optics is in general easier;

- there is not shadow cast on the module.

Lenses or mirrors are the primary optics, the first optical component met by the sunlight. After them a secondary optical element is necessary to homogenize the beam and to increase angular tolerance of the module.

The primary importance of having a secondary optics as a homogenizer is that the efficiency of the cell is decreased if the cell's surface is not illuminated in an uniform way. In fact if a part of the cell has a different power density, horizontal idle currents lower the power provided by the cell [5]. The actual losses depend on the ability of the cell to spread laterally the over generated current [6]. A similar effect is caused by chromatic aberration, because the different junctions are activated by different parts of the spectrum: if different parts of the solar spectrum take different paths, the nonuniformity of the spectrum over the cell's surface results in losses that degrade the performance of the cell.

The angular tolerance of the module is defined as the maximum misalignment angle from the direction of the Sun's rays at which the power from the module is still $90 \%$ of the power under perfect alignment (pointing to the Sun). This parameter is usually called the "angular acceptance". A large value of this is needed to compensate for mechanical misalignments, wind, tracker errors in pointing, and so forth. A typical value for highconcentration photovoltaics modules is a cone of half-angle 0.1 degrees.

14. Triple-junction solar cells. - Concentrating photovoltaics modules nowadays use almost only triple-junction solar cells. Triple-junction solar cells consist of three subcells (as seen by the solar light: top, middle and bottom), epitaxially grown monolithically. The subcell are connected in series using tunnel junctions (fig. 4).

Every subcell converts a part of the solar spectrum. The three subcells are seriesconnected. This allows at the same time high current and high voltage, both concurring to the final power that can be extracted from the cell.

In fact, using a mono-junction solar cell, if the energy gap of the junction is low a large amount of photons will be converted into electrical current: the current will be high, but the voltage low. Power is the product of current and voltage, current will then be low. This means that the energy of short-wavelength photons will be lost. On the other hand, if the energy gap is large, only shorter wavelength photons will be converted, voltage will be high but current low. This means that the lower energy photons will be wasted (their energy will only heat up the solar cell). A way to overcome this problem is to use multi-junction solar cells: in this way lower energy photons generate current (and low voltage) in the lower bandgap junction, and so forth up to the highest bandgap junction that generates power from the higher energy (shorter-wavelength photons), see fig. $5[7]$. 
Solar spectrum converted by each junction

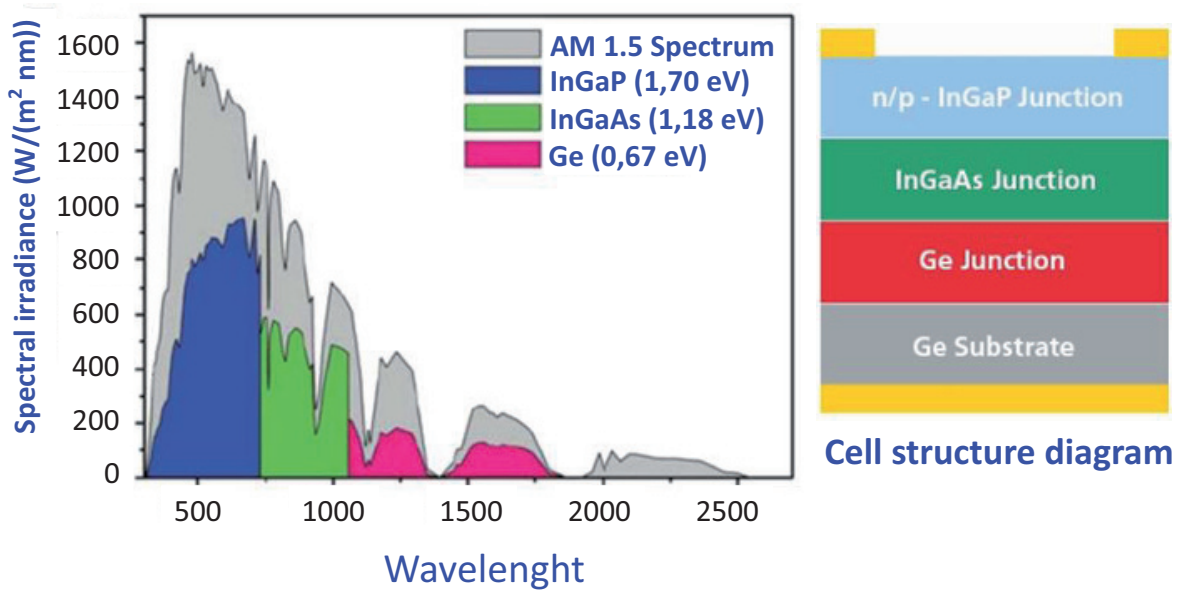

Fig. 4. - The triple junction (3J) solar cell is made of three subjunctions, each converting a part of the solar spectrum into electrical power.

\section{The portion of solar spectrum converted} depends on energy gap:
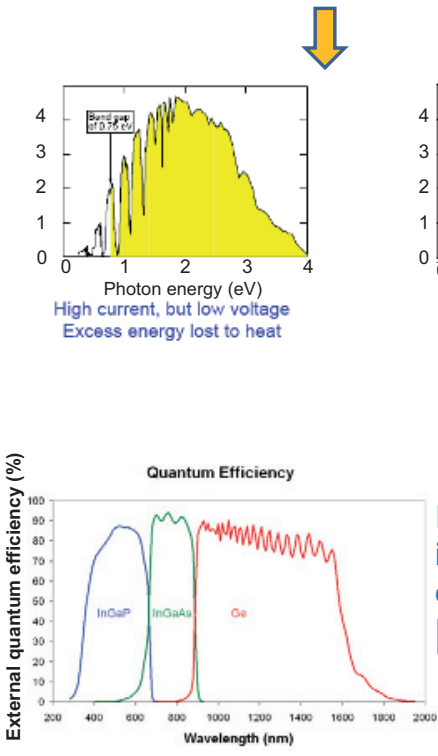

Silicon
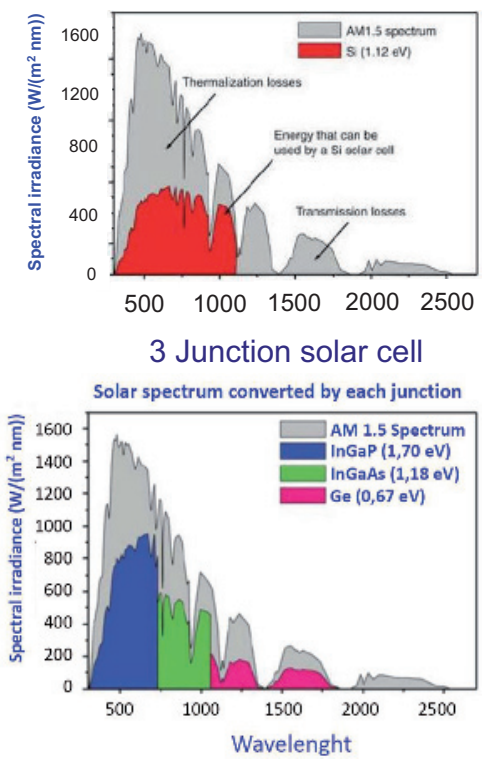

Fig. 5. - Up left: the case for a mono-junction solar cell. On the left, a lower bandgap junction, on the right higher bandgap junction ( $x$-axis in $\mathrm{eV}$, high energy on right of scale). In the first case the current is high and voltage is low, in the second voltage is high and current is low. In concentrating photovoltaics the use of multijunction solar cells allows to overcome this problem, having at the same time high voltage and high current. Right plots: upper panel, solar spectrum and energy converted by silicon solar cell; lower panel, solar spectrum and energy converted by 3J solar cell. Adapted from [7]. 
For a multiple junction solar cell, three parameters are of great importance:

- $V_{\text {oc }}$, the open circuit voltage,

- $I_{\mathrm{sc}}$, the short circuit current,

- FF, the fill factor.

These parameters have some dependences on concentration ratio and cell's temperature that must be taken into account when designing a concentrator [8].

The open circuit voltage has a logarithmic dependence on concentration ratio, and a linear dependence on temperature:

$$
V_{\mathrm{oc}}=\frac{n k T}{q \log (e)} \cdot \log (X)+V_{\mathrm{oc}, X=1}
$$

where $n$ is the diode ideality factor, $k$ Boltzmann's constant, $T$ the cell's temperature, $X$ the concentration ratio, defined as the ratio between the Module and the 1 Sun shortcircuit current. The formula is valid for $e^{\frac{q V_{\mathrm{oc}, X=1}}{k T}} \gg 1, \frac{1}{X}$.

$I_{\mathrm{sc}}$, the short circuit current, is linear with irradiation, in conditions that the cell is illuminated in a homogeneous way, that the solar spectrum does not change, and that the cell's temperature be constant.

The fill factor depends on cell's temperature and uniformity of illumination.

1\%.1. Dependence of efficiency on solar spectrum. A measurement of efficiency of a CPV module in principle should show a constant value over time, provided that air temperature and wind are constant. This not always happens, so one effect must be taken into account to explain deviations from this behaviour, one example of this being shown in fig. 6 .

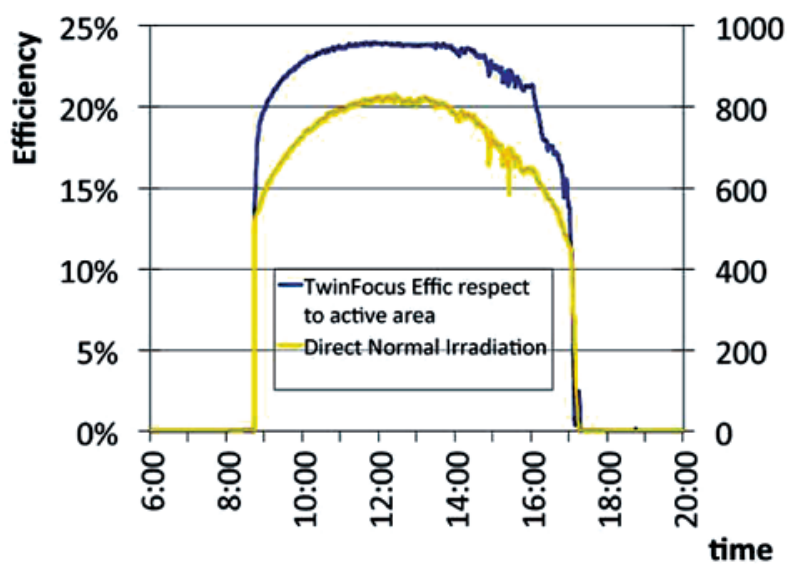

Fig. 6. - The efficiency of a CPV module over one day. The efficiency is not constant over the day, and the deviations cannot be traced to thermal effects. It can be seen that the efficiency is lower at beginning and end of the day. 


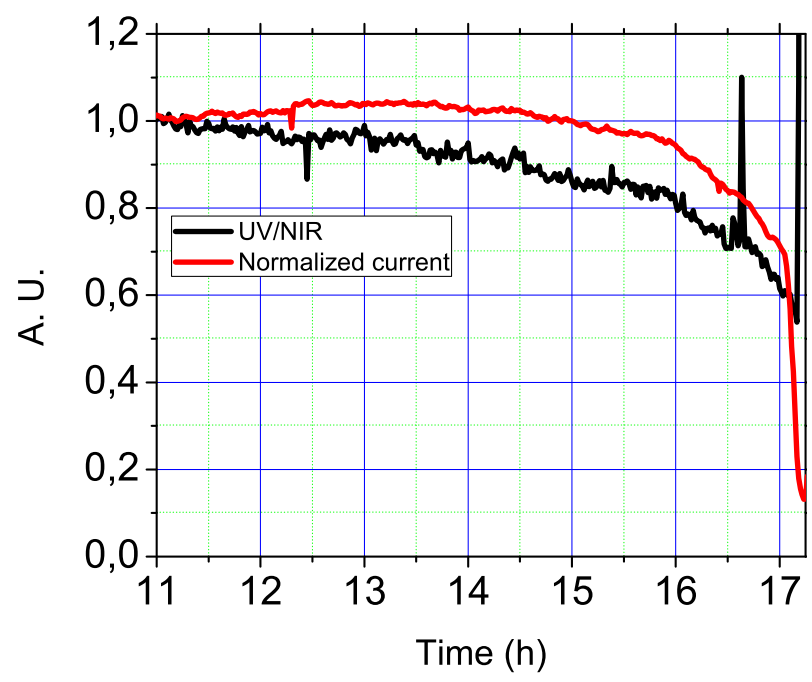

Fig. 7. - A measurement, performed in Northern Italy, of the short-wavelength (UV) and longwavelength (NIR) parts of the spectrum, together with the normalized current (current divided by DNI) generated. It is evident that when the UV part of the spectrum decreases relative to the NIR, also the current decreases. This is due to limiting of cell's current done by the top cell. The mismatch between decrease of UV/NIR and decrease of current is due to the fact that the UV and NIR measurements are performed only in a part of the spectrum and not in its whole.

One of the reasons why the efficiency is not constant is that the triple-junction solar cells are matched to the AM1.5 spectrum. At beginning and end of the day the solar spectrum shows a reduced ultraviolet (UV) component. In this condition the top cell, responsible for the conversion of higher energy photons, produces less current, limiting the current output of the middle and bottom cells, connected to it in a series. This is shown in fig. 7 [6].

Another consequence of this is that not only the efficiency strongly depends on solar spectrum (that is: location of the installation, date and time), but also the light must be concentrated in a way that there is not local current mismatch between the three subjunctions. This can be a problem especially using lenses for the concentrating optics: the chromatic aberration can result in higher local concentration ratio (a peak in concentration, higher than the average of the cell) in a subcell than in another one. This has as a consequence idle horizontal currents that spoil the efficiency of the cell.

15. Thermal management. - When designing a concentrated photovoltaics module, great attention must be paid to the thermal management: thermal management of CPV modules is one of the most important parts of its technology: the efficiency of the solar cells used decreases linearly increasing its temperature, at a rate of $-0.06 \% / \mathrm{K}$, due to electron-phonon interactions [9].

As in any solar cell, the efficiency of the cell is strongly dependent on the cell's temperature [8]. The open-circuit voltage of the $3 \mathrm{~J}$ solar cell decreases at a rate of 
$-4.5 \mathrm{mV} / \mathrm{K}$, and the short-circuit current increases at a rate of $+4.7 \mathrm{~mA} / \mathrm{K}$ (over a surface of $1 \mathrm{~cm}^{2}$ ). The two effects do not cancel out: power is neither extracted at short circuit nor at open circuit, but in an intermediate point, called the maximum power point. The value of this point is given by the fill factor, which decreases at increasing temperature, so that the overall effect of the temperature rise is: $-0.06 \% / \mathrm{K}$.

More precisely, the rate of decrease of the open-circuit voltage also depends on irradiation, being smaller at higher irradiation. This effect helps when considering higher concentration factors. Moreover, higher concentration factors result in higher voltages (at the same temperature), so that with a well-designed heat sinking one can compensate the higher thermal power to be dissipated (potential higher temperature) with a higher voltage (it means higher power) that allows a more tolerant design.

For a review of the different ways to manage heat, see [10] and [3]. Basically, a point focus concentrator (where the focussed light from the Sun is in a position separated in a matrix) can be constructed with passive cooling (air cooling, not fan forced), where linear focus and heliostat require active cooling or even water cooling.

1*6. Tracking issues. - As CPV concentrators use only the direct part of the solar radiation, CPV modules must be mounted on a structure able to track the motion of the Sun. For medium- and high-concentration modules a dual-axis tracker is needed. There are basically two main types of trackers: pedestal, having a central column bearing one or two "wings" containing the modules, or Azimuth-altitude dual-axis tracker, in which the modules rotate on a metal or concrete circular track for tracking east-west, and have a second motor to control the elevation. The advantage of the pedestal configuration is the possibility of using the land under the tracker for instance for agriculture, and in general the possibility to mount larger areas of modules. The second configuration has a lower profile, which is an advantage for maintenance and for less visual impact.

For tracking, usually a hybrid feedback control is used: the coarse tracking is granted by the use of a microcontroller using an equation that has the date, time, altitude and longitude as inputs, and the position of the Sun as output. The microcontroller is then able to convert the position of the Sun into the position of the two motors. The fine tracking is then controlled by a closed-loop feed-back, using the difference in illumination in some photosensors as error signals.

For concentrating photovoltaics with high concentration a tracking precision no worse than $0.1^{\circ}$ is needed. In fact, as seen in sect. 1:3 the angular acceptance of a HCPV optics usually is of about $1^{\circ}$. It must cover for the $0.26^{\circ}$ of Sun's subtended angle, and for mechanical misalignment on mounting, and for wind action. The sum of all these angles gives the acceptance angle needed. As the acceptance angle is defined by the optics, the tracker must be precise: the time spans in which the misalignment is over the angular acceptance the CPV modules are not producing energy.

Another important aspect of solar trackers is their ability to operate under windy conditions. To avoid that the tracker and the modules are damaged during very windy days, a control is implemented that puts the tracker in stow position. The lower the value of wind speed is used as a threshold, the larger the energy production is wasted. 


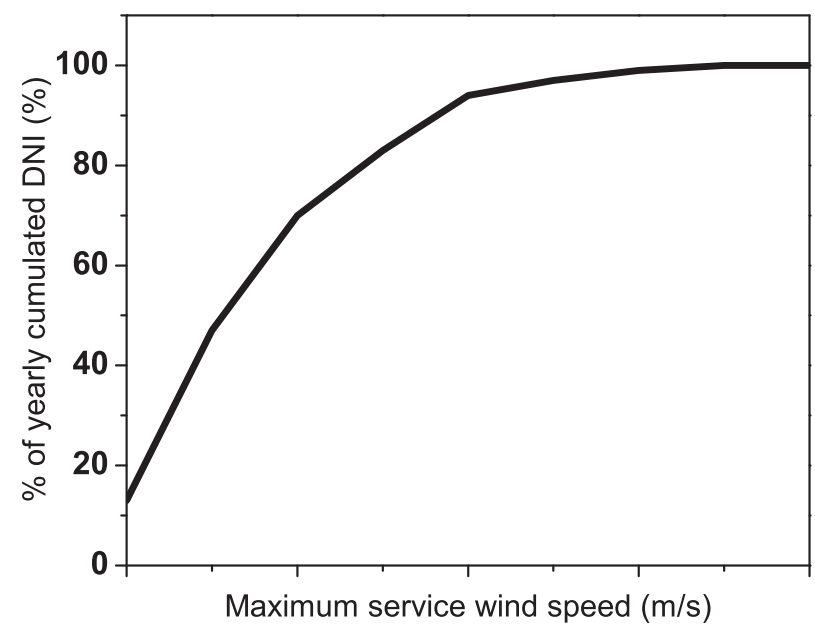

Fig. 8. - Yearly cumulated DNI (Direct Normal Irradiance) as a function of wind speed. Adapted from [11].

Nevertheless a compromise must be accepted, since a too high threshold may cause the tracker to work under too severe wind conditions, resulting in damages [11]. Unfortunately increasing the maximum wind speed at which the tracker can still active (not in secure stow position and within precision range) results in higher costs, so it is necessary to define how much DNI will be present in average when wind speed is over the threshold value. This can be seen in fig. 8 and fig. 9. The solar tracker is one of the main costs of a CPV system, thus to save the overall cost of the power plant it is necessary to design the tracker taking into account the yearly cumulated DNI as a function of the wind speed for that location, and the cost of the tracker as a function of its wind speed threshold. So proper design of the tracker must follow a computation of the levelized cost of energy (LCOE), defined as the cost of the plant divided by the energy output of the plant.

1'7. Rated power-real power. - Compared to silicon-based solar modules, rated power of CPV modules based on 3J solar cells is nearer to effective power. This is due primarily to the fact that the dependence of efficiency on temperature is almost ten times lower for 3J. Usually the values given are a ratio of $84 \%$ between actual power and rated power for mono-Si photovoltaic modules, and $96 \%$ for CPV [7]. For silicon photovoltaics both rated (STC) and in-field (NOCT) power are well defined by IEC standard, in CPV only recently it appears to be a convergence on values corresponding to lab and field: CSTC (Concentrator Standard Test Conditions) with $1000 \mathrm{~W} / \mathrm{m}^{2}$ and cell temperature $25^{\circ} \mathrm{C}$, CSOC (Concentrator Standard Operating Conditions) with $900 \mathrm{~W} / \mathrm{m}^{2}$ and $20^{\circ} \mathrm{C}$ air temperature.

1'8. Where: DNI resources. - Due to the fact that the concentrating photovoltaics modules use lenses or mirrors, they can only cope with rays that have a precise direction: the normal of the module surface must point to the Sun. For this reason a concentrating 


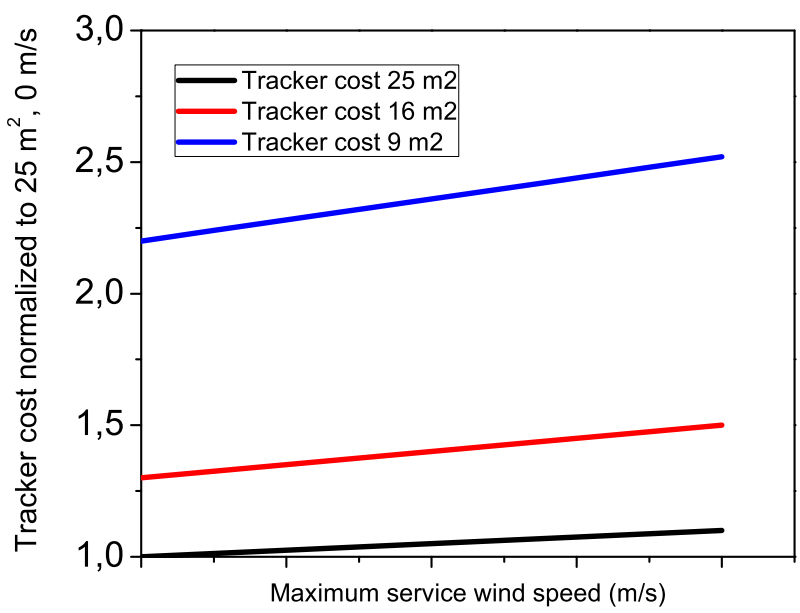

Fig. 9. - Normalized cost of a tracker, as a function of the maximum wind speed at which it can operate, in arbitrary units. Adapted from [11].

photovoltaics module produces electrical power only using the direct normal irradiation (DNI). This means that the modules do not use all the solar resource (the global irradiation is made of the normal, also called "beam", and the diffuse, that is not used by the CPV module). The fact that a CPV module tracks the Sun increases the irradiation impinging the module. Thus, when deciding whether to install a CPV module or a flat (not moving) module the parameter that must be taken into account is not the installed power but the produced energy and so its final cost.

The procedure is then to obtain reliable information about DNI on the site of interest, calculate the amount of energy that can be produced using the module, and find out the final cost of this energy. By doing this operation all costs must be included, i.e. installation costs and financial costs. By dividing energy by the real (actualized) cost one obtains the LCOE (levelised cost of energy). This is the value that must be compared when choosing between different energy systems. Performing this operations it is soon clear that a CPV module is not convenient in a low DNI region, but can be financially competitive in high DNI and high-average-temperature regions.

1`9. Conclusions. - Concentration photovotlaics (CPV) is a technology to create highefficiency solar modules. It is based on the principle of reducing semiconductor's surface by the use of molded plastic and aluminum. The use of high-efficiency (40\%) triplejunction solar cells allows concentration factors up to $2000 \times$, and a module efficiency up to $30 \%$. This efficiency can be increased by a careful use of the state-of-the-art technologies, above all in the files of non-imaging optics and heat sinking.

In high DNI and high-average-temperature regions CPV modules can deliver more yearly energy than conventional flat photovoltaics modules. 
This work is supported by the Centro Studi e Ricerche "E. Fermi", Rome (Italy) and by the Polo Fotovoltaico Veneto http://www.polofotovoltaicoveneto.it/.

\section{REFERENCES}

[1] Swanson R., Progr. Photovolt. Res. Appl., 8 (2000) 93.

[2] Winston R., Miñano J. C. and Benítez P., Nonimaging optics (Elsevier) 2005.

[3] Royne A., Dey C. J. and Mills D. R., Sol. Ener. Mater. Sol. Cells, 86 (2005) 451.

[4] Tech. Rep. IEC 62108, IEC (12 2007).

[5] Kurtz S. R., Faine P. and Olson J. M., J. Appl. Phys., 68 (1990) 1890. URL http://link. aip.org/link/?JAP/68/1890/1

[6] Domínguez C., Victoria M., Herrero R., Askins S., Antón I. and Sala G., 8th International Conference on Concentrating PV Systems (CPV-8), Toledo (Spain) (2012).

[7] KurTz S., Technology overview, in CPV Boot Camp (NREL) 2010.

[8] Kinsey G. S., Hebert P., Barbour K. E., Krut D. D., Cotal H. L. and A. S. R., Prog. Photovolt: Res. Appl., 16 (2008) 503.

[9] Morais R. R. O., Dias I. F. L., Duarte J. L. and Laureto E., Braz. J. Phys., 40 (2010) 15.

[10] Luque A., Sala G., Miñano J. C. and Benítez P., Sol. Ener. Mater. Sol. Cells, 51 (1998) 269.

[11] Luque-Heredia i., Quéméré G., Cervantes R., Laurent O., Chiappori E. and Chong J., The sun tracker in concentrator photovoltaics, in Next Generation of Photovoltaics, edited by Cristóbal López A. B., Martí Vega A. and Luque López A., Springer Ser. Opt. Sci., Vol. 165 (Springer Berlin Heidelberg) 2012, pp. 61-93. 